Ophthalmologe 2020 $\cdot 117: 963-964$

https://doi.org/10.1007/s00347-020-01131-4

(c) Springer Medizin Verlag GmbH, ein Teil von Springer Nature 2020

Robert P. Finger

Universitäts-Augenklinik Bonn, Bonn, Deutschland

\title{
Künstliche Intelligenz in der Augenheilkunde
}

Im Bereich der künstlichen Intelligenz (KI) sind - wie bei allen neuen Möglichkeiten, Techniken und Verfahren die Erwartungen an die leistbaren Verbesserungen durch deren Einsatz sehr hoch. Gleichzeitig entstehen Befürchtungen, dass konventionelle Arbeitsschritte überflüssig werden und sich somit der humane Arbeitsbedarf reduziert. Auch in der Augenheilkunde haben Anwendungen, die auf KI beruhen, bereits Einzug gehalten. Die Segmentierung von OCT(optische Kohärenztomographie)-Schichten zur Beurteilung von z. B. Veränderungen in der Dicke einer oder mehrerer Schichten oder die Auswertung von Fundusbildern zur Erkennung von Anzeichen einer diabetischen Retinopathie durch z. B. das FDA(Food and Drug Administration)-zugelassene IDxDR sind nur einige wenige dieser bereits existierenden Anwendungen.

\section{》) In der Augenheilkunde haben Anwendungen, die auf KI beruhen, bereits Einzug gehalten}

Um einen besseren Überblick darüber zu ermöglichen, was von KI - insbesondere in der Augenheilkunde - überhaupt zu erwarten ist, wie man beurteilen kann, ob der Einsatz sinnvoll, die Ergebnisse glaubwürdig und in die Behandlungsrealität übertragbar sind und wie ein möglicher zukünftiger Arbeitsalltag in der Augenheilkunde mit mehr Hilfssystemen aus dem Bereich der künstlichen Intelligenz aussehen könnte, haben wir dies in den 4 Artikeln dieses Leitthemas genauer beleuchtet.

Im ersten Beitrag von Morelle et al. wird knapp und allgemeinverständlich dargestellt, was die Grundlagen der KI sind und wie diese auf die automatische Bildauswertung angewandt werden können [1]. Der Vorteil der multimodalen gegenüber der unimodalen Bildgebung für die Erhöhung der Sicherheit von Diagnosen oder zur Beurteilung von Verläufen ist im klinischen Alltag unstrittig. Diese lässt sich bislang aber nur unzureichend in den verfügbaren KI-Lösungen umsetzen, die fast ausschließlich auf unimodalen Ansätzen basieren. Hier gibt es aber bereits Ansätze, auch multimodale KILösungen zu etablieren.

Nach diesem Überblick über die methodischen Grundlagen der KI und deren Anwendung im Bereich der augenheilkundlichen Bildgebung diskutieren Pfau et al. in ihrem Beitrag, wie man KI-Studien strukturiert in Bezug auf ihre Qualität, die Verlässlichkeit der Ergebnisse sowie deren Übertragbarkeit außerhalb der jeweiligen Studie beurteilen kann [2]. Dies ist sehr hilfreich, da bislang ein Leitfaden hierzu in der Augenheilkunde fehlt und ganz konkrete Punkte an die Hand gegeben werden, anhand derer man die Güte einer KI-Studie bewerten kann.

Im Folgenden stellen Treder et al. verschiedene KI-Studien vor, die Lösungen für das Erkennen, die Quantifikation und Veränderungen über die Zeit bei Makulaödemen (MÖ) entwickelt und erprobt haben [3]. Verschiedene Studien bauen auf der automatisierten Bildanalyse auf und haben einen Algorithmus angeschlossen, der eine Behandlungsempfehlung (z. B. Behandlung einer MÖ-Zunahme) ermöglicht. Treder et al. stellen hier jedoch den unbeaufsichtigten Einsatz in der klinischen Versorgung infrage, da durch Fehlentscheidungen des Algorithmus verheerende Folgen entstehen könnten. 
Im abschließenden Beitrag des Leitthemas beleuchten Kern und Kortüm umfassend, wie die klinische Routine unter Einsatz von KI in der nicht allzu fernen Zukunft im Jahr 2030 aussehen könnte [4]. Eingegangen wird in diesem Beitrag aber auch auf die zu erwartenden Verbesserungen in der verfügbaren Bildgebung, telemedizinische Dienstleistungen und virtuelle Kliniken, die unabhängig von bzw. zusätzlich zu KI eingesetzt werden können und so den Arbeitsalltag verändern werden.

Zusammenfassend gibt das Leitthema einen spannenden Überblick über Grundlagen der KI sowie Qualitätsanforderungen und Anwendungsbeispiele. Im Rahmen der zunehmenden Digitalisierung nicht nur unserer Arbeitswelt, sondern unseres gesamten Lebens ist es sehr wahrscheinlich, dass wir zunehmend mit KI im Alltag konfrontiert werden. Dies birgt Chancen, die bei einem selbstbestimmten Einsatz in der und durch die Augenheilkunde das Potenzial haben, die augenheilkundliche Versorgung und den augenärztlichen Arbeitsalltag zu verbessern.

Ihr Prof. Dr. Robert P. Finger

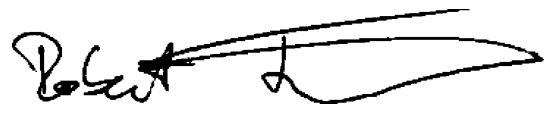

\section{Korrespondenzadresse}

Prof. Dr. Robert P. Finger,
PhD
Universitäts-Augenklinik
Bonn
Ernst-Abbe-Str. 2,
53127 Bonn, Deutschland
robert.finger@ukbonn.de

Interessenkonflikt. R.P. Finger gibt an, dass kein Interessenkonflikt besteht.

\section{Literatur}

1. Morelle O, Wintergerst M, Finger RP (2020) Multimodale Bildgebung und -auswertung im Zeitalter von Künstlicher Intelligenz. Ophthalmologe. Im Druck

2. Pfau $M$, Walther $G$, von der Emde $L$ et al (2020) Künstliche Intelligenz in der Augenheilkunde: Leitfaden für Ärzte zur kritischen Bewertung von Studien. Ophthalmologe. Im Druck

3. Treder M, Diener R, Eter N (2020) Künstliche Intelligenzzum Management von Makulaödemen
Ophthalmologe. https://doi.org/10.1007/s00347020-01110-9

4. Kern C, Kortüm K (2020) Netzhautsprechstunde im Jahr 2030. Ophthalmologe. https://doi.org/10. 1007/s00347-020-01111-8

\section{Terminankündigung}

\section{1}

AAD 2021

Düsseldorf, 16.03. - 20.03.2021

Kongressorganisation: Berufsverband der Augenärzte e.V. (BVA)

http://www.aad.to/

DOG 2021

Berlin, 30.09. - 03.10.2021

https://www.dog.org/

2022

AAD 2022

Düsseldorf, 15.03. - 19.03.2022

Kongressorganisation: Berufsverband der Augenärzte e.V. (BVA)

http://www.aad.to/

DOG 2022

Ort noch in Absprache, 29.09. - 02.10.2022 https://www.dog.org/

2023

AAD 2023

Düsseldorf, 21.03. - 25.03.2023

Kongressorganisation: Berufsverband der Augenärzte e.V. (BVA)

http://www.aad.to/

DOG 2023

Berlin, 28.09. - 01.10.2023

https://www.dog.org/ 